\title{
From Barraquer-Simons syndrome to treatment perspectives for obesity and diabetes mellitus - a case report
}

\author{
Ancuta Gabura ${ }^{1}$, Laura Ciobanu ${ }^{*, 1,2}$ \\ 1 "Grigore T Popa" University of Medicine and Pharmacy lasi, Romania, ${ }^{2}$ Clinical Hospital of \\ Rehabilitation, lasi, Romania
}

\begin{abstract}
Introduction: Barraquer-Simons syndrome, also called acquired partial lipodystrophy (APL), is a rare form of progressive lipodystrophy with no more than 255 cases reported so far.

Case report: We present a 68-year old female, diagnosed with situs inversus totalis at 7 and nodular goiter at 14 years old. One year later, due to progressive loss of subcutaneous fat in the upper part of the body, facial fat atrophy and pseudohypertrophy of the muscular mass, she was diagnosed with Barraquer-Simons syndrome. She is associating numerous metabolic (diabetes mellitus, hypertriglyceridemia, hepatic steatosis), cardiac (high blood pressure, ischemic coronary disease with stable angina pectoris and heart failure class III NYHA) and immuno-rheumatological (polymyositis, rheumatoid arthritis with osteoporosis) comorbidities.

Discussion: Studying similar cases, scientists have observed that patients with APL have metabolic diseases such as severe insulin resistance, dyslipidemia, hepatic steatosis, which are also common for obese patients. This correlation has led to a shift in the current thinking about metabolic disease from a 'glucocentric' towards a more 'lipocentric' view, with fat perceived as an essential organ in the metabolic homeostasis maintenance. Therefore, the adipocyte hormones as leptin or adiponectine have become targets in the new treatments of diabetes and obesity.

Conclusion: The study of rare diseases has brought valuable therapy perspectives such as Trodusquemine (an enzime that regulates insulin and leptin signaling pathways), AdipoRon (adiponectine receptors agonists) and fat transplantation. If they pass the clinical trials, they might be considered a therapeutic approach for our patient in the future.
\end{abstract}

Keywords: Barraquer-Simons syndrome, adipokine, obesity, diabetes miellitus

\section{Introduction}

The Barraquer-Simons syndrome, APL or cephalothoracic lipodystrophy, was described by the Spanish physician Barraquer in 1906 and the German physician Simons in 1911 [1].

Received: August 2014; Accepted after review: September 2014; Published: September 2014

*Corresponding author: Laura Ciobanu, Assoc Prof, MD, PhD, FERS; Clinical Hospital of Rehabilitation, 14, Pantelimon Halipa St, 700661, Iasi, Romania. Email: lauraciobanu2012@gmail.com
There were reported only 255 cases since then. It is characterized by progressive and symmetrical loss of adipose tissue in craniocaudal direction, starting with the face and progressively involving the trunk, arms and legs up to the thighs. However, arms and legs are rarely affected.

The adipose stores of the gluteal regions and lower extremities (including soles) tend to be either preserved or increased, particularly among women [2]. 
The onset of the disease often occurs in the first and second decade of life, predominantly affecting females, with a ratio of $4: 1$ to males [3].

The determinant cause of BarraquerSimons disease has not yet been elucidated. The only aspect it has been agreed on is that it is not an inherited disorder in the classic mendelian sense; it rather represents a phenotype with a complex etiology [4].

\section{Case report}

We present the case of TDE, a female patient of 68-year old with Barraquer-Simons disease diagnosed in the second decade of life. The patient is the fourth child of healthy nonconsanguineous parents. During the first 3 months of pregnancy, her mother drunk an antimalaric substance called chinin in the attempt to abort the baby. The attempt failed and she was born apparently healthy.

At the age of 7 she was diagnosed with situs inversus totalis during a chest radiological checkup required for entering the first grade. At 15 years old the loss of fat from the body upper part was already visible and she was diagnosed with nodular goiter and APL. Severe systemic hypertension was found at 32 years of age and idiopathic hepatitis at 35 . At 61 she already had diabetes mellitus type 2 , being on insulin in the last three years.

At 65-year old doctors have found her with positive serum markers for reumathoid artrithis and she was also diagnosed with osteoporosis based on DEXA scan and polymiositis. A muscle disease was suggested by her sudden partial painful contractions to the abdomen or limbs, especially to the lower ones, so she suffered a muscle biopsy and the diagnosis was issued based on the pathological study.

The patient had 6 pregnancies, with 4 abortions ( 2 natural and 2 required ones) and 2 children, normally delivered and breast feeded; she has now 5 grandchildren, all in good health. The family medical history is irrelevant, none of her siblings having been diagnosed with Barraquer-Simons disease or other metabolic conditions. She worked as a farmer and denies smoking or drinking alcohol.
TDE is a chronic patient of the Clinical Hospital of Rehabilitation in lasi. Her last admittance was in September 2013 for shortness of breath, productive coughing with mucopurulent sputum and generalized joint pain. Physical examination revealed loss of adipose tissue in the upper part of the body, with complete facial fat atrophy, pseudo hypertrophy of the muscular mass and hirsutism. Comparing Figures 1, 2 and 3, we can see that these features have progressed along the years.

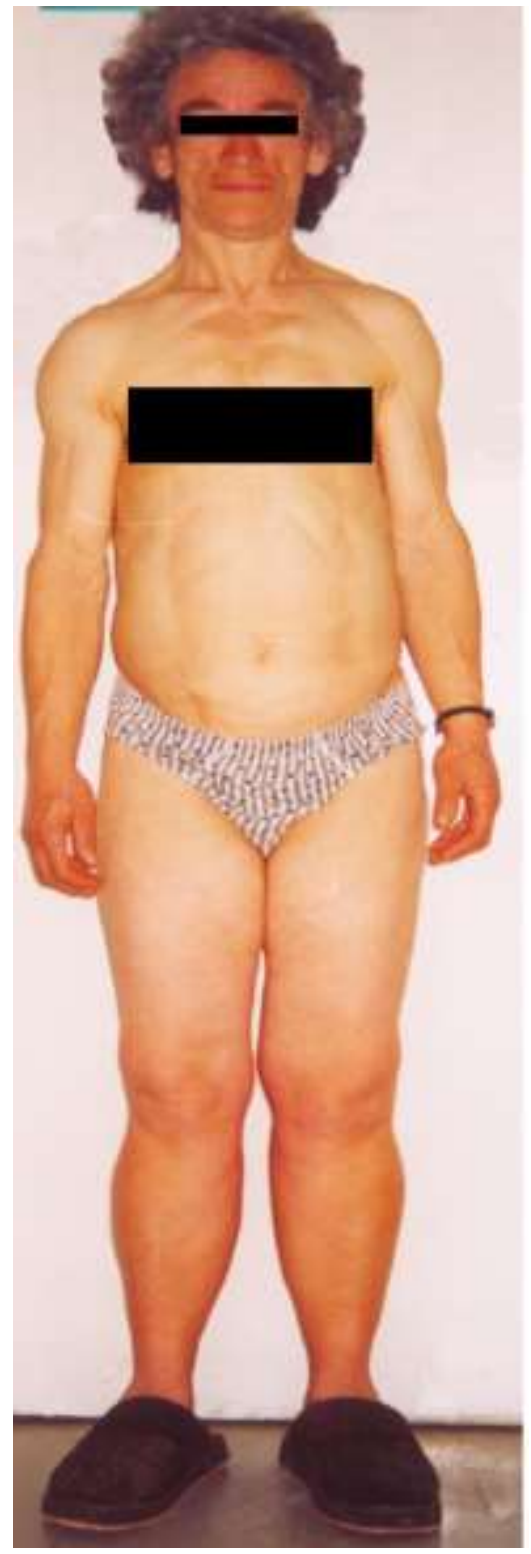

Fig.1. The patient at age of 54: loss of subcutaneous fat in the upper part of the body, with facial fat atrophy, pseudohypertrophy of the muscular mass and facial hirsutism. 


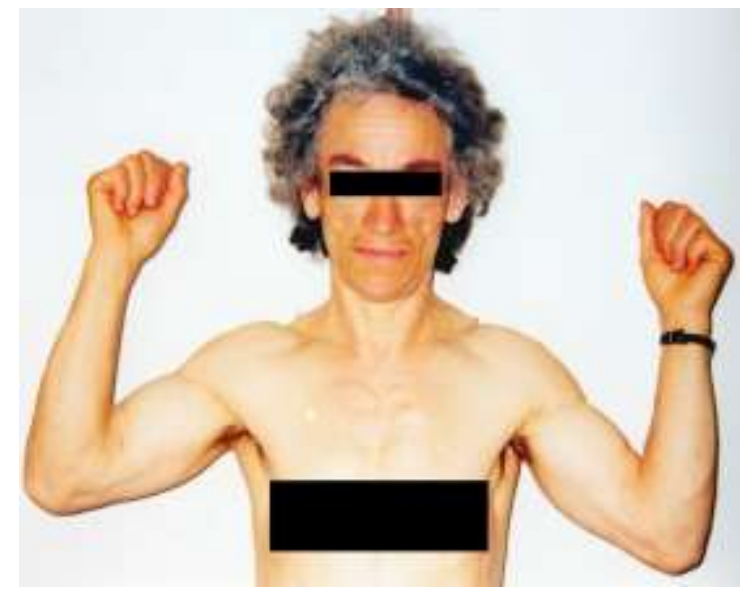

Fig. 2. The patient at the age of 60 : significant muscle pseudohypertrophy and disappearance of Bichat's fat pad

Examination of the articular system has revealed joint pains mainly in the cervical and sacro-iliac region, painful hands and feet, especially in the night, with decreased range of motion and characteristic deformities for rheumatoid arthritis; the Patrick maneuvers were bilateral positive.

Thorax examination showed bilateral basal dry crackles and dullness to the left base. Systemic blood pressure was around 140/70 $\mathrm{mmHg}$ and heart rate 60 beats/min. A strong systolic heart murmur was audible in all areas of auscultation with a maximum of $6 / 6$ intensity in the mitral and aortic areas.

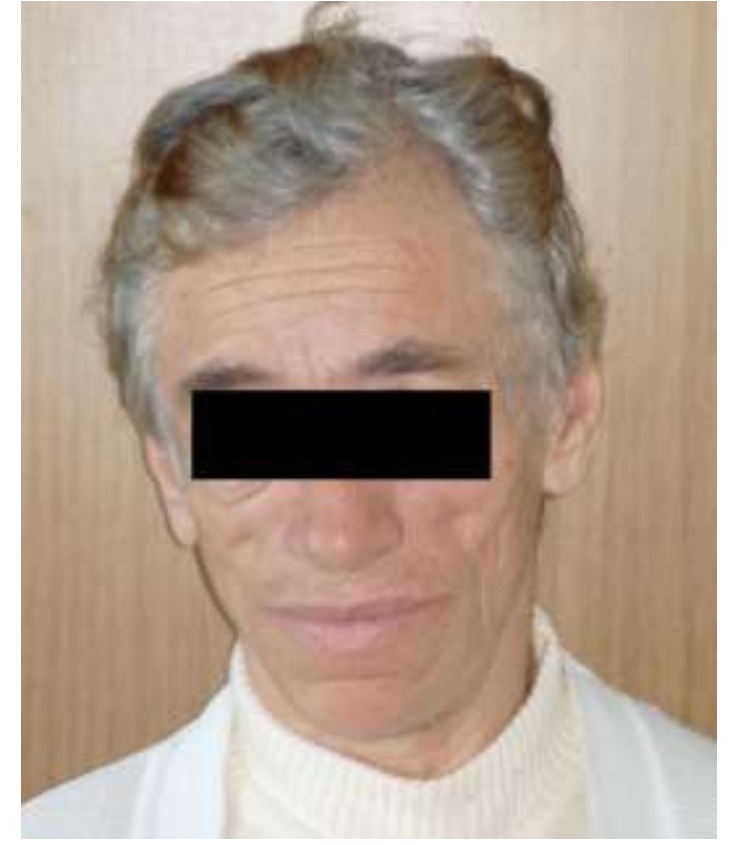

Fig. 3. Patient at age of 65: significant loss of subcutaneous fat including the Bichat's fat pad

The liver was palpable at $10 \mathrm{~cm}$ under the left costal margin and the spleen at $3 \mathrm{~cm}$ under the right one. The liver and spleen were spontaneously and at palpation sensitive.

The pathological results of laboratory tests are shown in Table 1. Normal range values are delivered by the Rehabilitation Clinical Hospital's Laboratory.

Table 1. Laboratory tests showing lower HDL-cholesterol and elevated glucose and triglycerides levels

\begin{tabular}{lcc}
\hline Blood test & Value & Normal range \\
\hline Hematocrit (\%) & 36.53 & $38-47$ \\
VSH (mm/h) & 15 & $10-12$ \\
$\begin{array}{l}\text { HDL-cholesterol } \\
\text { (mg/dl) }\end{array}$ & 35.6 & $40-60$ \\
\hline $\begin{array}{l}\text { Triglycerides (mg/dl) } \\
\text { Glucose (mg/dl) }\end{array}$ & 339.55 & $0-150$ \\
\hline
\end{tabular}


The ECG registration on the right hemithorax placement (Figure 4) has shown a right QRS axis deviation at 120 degrees, an important left ventricular hypertrophy with a Sokolow Lyon index of $86 \mathrm{~mm}$ (in the precordial leads, there is a special registration with $5 \mathrm{~mm}$ standing for $1 \mathrm{mV}$ ) and inverted $\mathrm{T}$ waves from V3 to V6, as well in DIII and aVF. The chest Xray showed dextrocardia, cardiomegaly, pulmonary congestion and aortic atheromatosis (Figure 5).

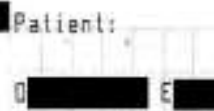

62 year II, 15

$\ldots \ldots \mathrm{kn} / \ldots \mathrm{kg}$
HR 62/ain Axis:

P $12 B$ 025119.

T -35 ,

RR 968 as

P. $120 \mathrm{~ns}$

$P R$ 20Q 95

QR5 $\quad$ B as

DT 462 as

QTC 471 as
P (II) $0.11 \mathrm{nW}$

5 (VI) $-n$

$R$ (U5) $5.51 \mathrm{nW} 5.62$

5okol. $9.71 \mathrm{nV}$
5LPRRUENTRICULAR ARRHYTHMIA

LEFT RTRIAL ABNORHALITY

ABNORNAL RIGHT AXIS DEUIATION

LUH UI TH REPOLARIZATION AENORHALITY

DR5(T) CONTOUR RENORHAL1TY

CONSTSTEXT WITH HIGH LATERAL INFARCT

AGE UNDETERHINED

62

UNCONF IRMED REPQRT
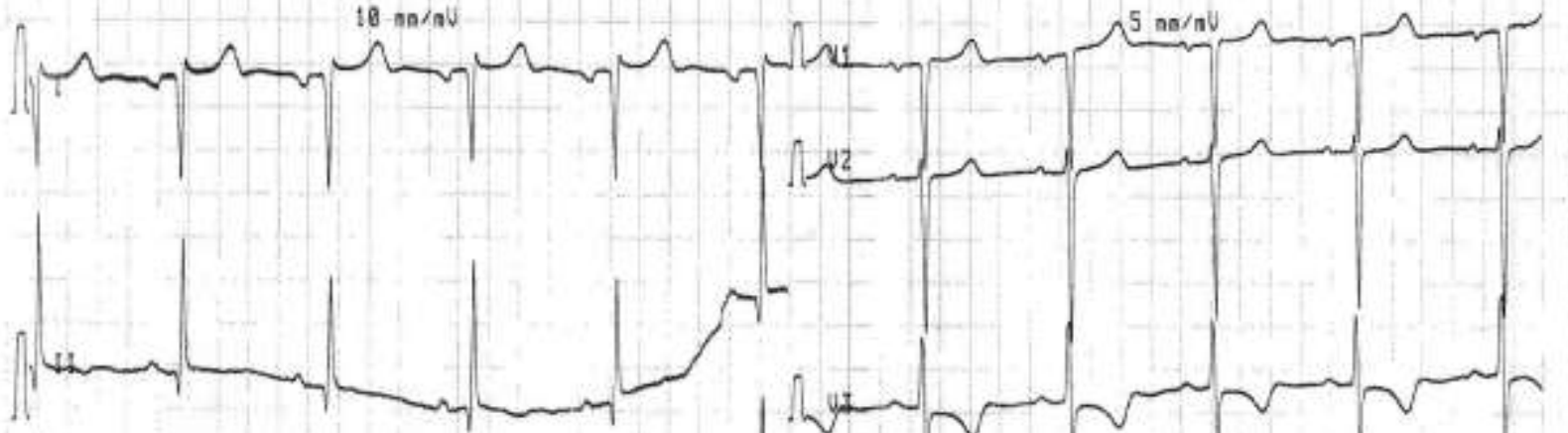

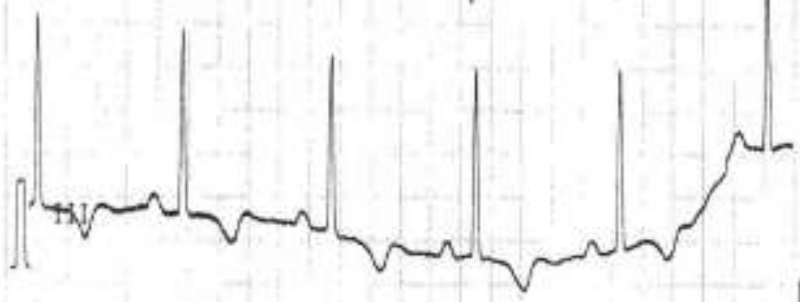

If
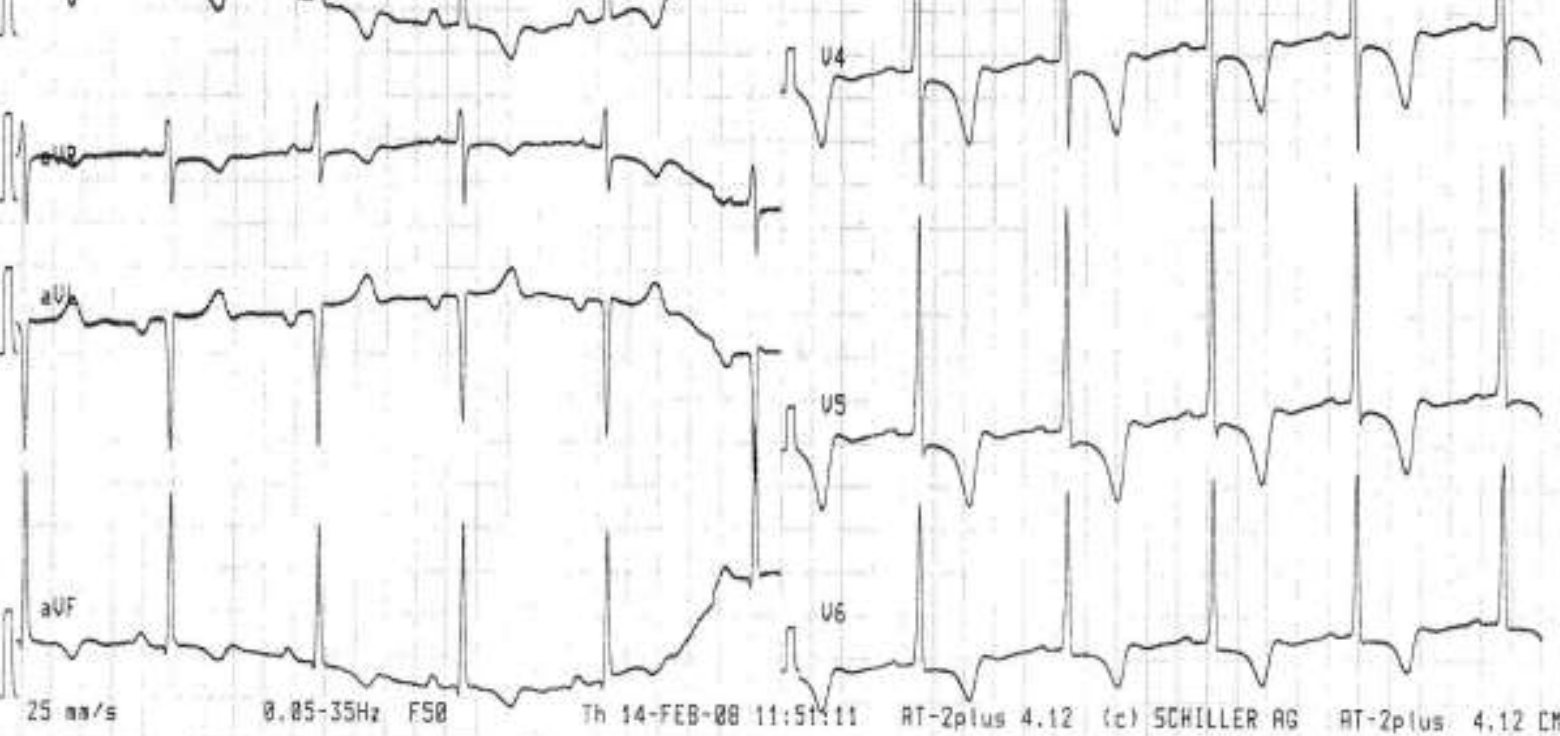

Fig. 4. ECG registration in right hemithorax position 


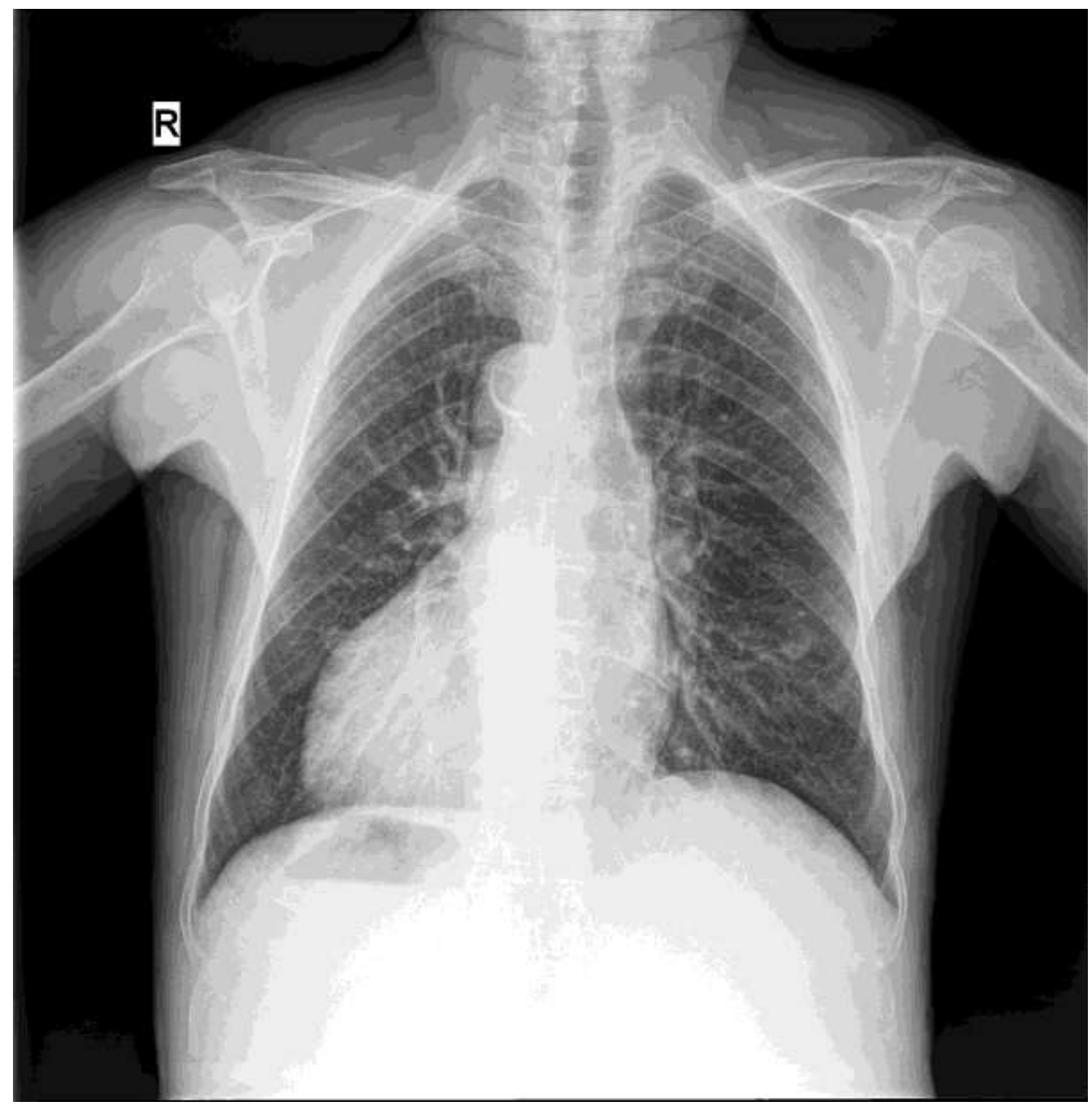

Fig. 5. The A-P chest X-ray

The abdominal ultrasonography revealed intense hepatic steatosis, small stones and bilateral double kidney, while the thyroid ultrasound examination discovered same old multiple nodules.

On release, the patient was diagnosed with the following: chronic bronchitis, situs inversus totalis, polinodular goiter, Barraquer-Simons syndrome, insulin-dependent type 2 non-obese diabetes complicated with polyneuropathie, hypertriglyceridemia, liver steatosis, essential hypertension stage 3 well drug controlled, obstructive hypertrophic cardiomyopathy, ischemic heart disease, heart failure class III NYHA, rheumatoid arthritis stage II, polymyositis, osteoporosis.

\section{Discussions}

Scientists found that both patients with APL and obese patiens have severe insulin resistance, dyslipidemia, hepatic steatosis and features of hyperandrogenism, as shown in the presented case. 
This illustrates a complex relationship between metabolic health and the fat mass. An explanation would be that adipocytes not only store fat but also secrete a variety of bioactive proteins into circulation.

These secretory proteins, which have been collectively named adipocytokines, include leptin, tumor necrosis factor (TNF)- $\alpha$, plasminogen-activator inhibitor type 1 (PAI-1), adipsin, resistin and adiponectin [5].

Leptin controls delivery of free fatty acids into adipocytes and limits the delivery of free fatty acids to nonadipocytes (liver and muscle cells), thus protecting nonadipocytes from lipotoxicity. Leptin deficiency results in an increase in exposure of muscle cells to free fatty acids and allows uptake of free fatty acids for oxidation and energy utilization, resulting in insuline resistance [6].

In studies done on human aortic endothelial cells, adiponectin has been shown to dosedependently decrease the surface expression of vascular adhesion molecules known to modulate endothelial inflammatory responses [7]. It also inhibits proliferation of vascular smooth muscle cells [8] and concentrates within the vascular intima of catheter - injured vessels [9]. In clinical studies, low adiponectin levels have been associated with an atherogenic lipid profile [5].

This theory is backed up by experimental work on mice. The peroxisome proliferatoractivated receptor gamma (PPARG) P465L mouse model is the equivalent human variant of partial lipodystrophy. PPARG is a member of the nuclear hormone receptor superfamily and is highly expressed in adipose tissue, monocytes, macrophages, colon and at lower levels in multiple other tissues. It plays a critical role in adipocyte differentiation.

These mice have APL features, but do not express insulin resistance unless crossed with severely hyperphagic leptin-deficient ob/ob mice. This shows the key role that leptin plays in the onset of diabetes.

Another experiment has used transgenic mice overexpressing adiponectin. When crossed with obese mice, these mice become even more obese and yet manifest significantly improved metabolic homeostasis, suggesting that if adipose tissue can expand to accommodate surplus calories, insulin resistance can be ameliorated [7].

Starting from these findings, treatment perspectives for metabolic diseases were developed. Some of them have passed clinical trials, like inhibitors of protein tyrosine phosphatase 1B (PTP1B), while others only showed efficiency on mice, like adiponectin natural stimulants, adiponectin receptors agonists or fat transplantation.

PTP1B regulates the insulin and leptin signaling pathways. Inhibition of this enzime controls appetite and blood sugar levels. The lead compound in this series is known as MSI1436 (trodusquemine) which completed in 2009 phase 1 of clinical trials and has demonstrated dose-dependent weight loss and glucose control even after a single dose [10].

An adiponectin natural stimulant is found in the turmeric plant. Curcumin is one of the principal anti-inflammatory and healthful components of turmeric comprising $2-8 \%$ of most turmeric preparations. Studies have shown that it induces the expression of adiponectin and also has effects to inhibit adipocyte differentiation and to promote antioxidant activities [11].

Synthetic adiponectin stimulants were developed as adiponectin receptors agonists. Adiponectin binds to adiponectin receptors AdipoR1 and AdipoR2, and exerts antidiabetic effects via activation of AMP-activated protein kinase (AMPK) and PPAR- $\alpha$ pathways, respectively.

The orally active synthetic small-molecule AdipoRon bounds to both AdipoR1 and AdipoR2 in vitro. AdipoRon showed very similar effects to adiponectin in muscle and liver, such as activation of AMPK and PPAR- $\alpha$ pathways, and ameliorated insulin resistance and glucose intolerance in mice fed with a high-fat diet, which was completely obliterated in AdipoR1 and AdipoR2 double-knockout mice.

Moreover, AdipoRon ameliorated diabetes of genetically obese rodent model $\mathrm{db} / \mathrm{db}$ mice, and prolonged the shortened lifespan of $\mathrm{db} / \mathrm{db}$ mice on a high-fat diet [12].

An alternative strategy to relieving metabolic stress on diseased adipose tissue would be to provide extra fat depots. 
In 2011, Dr. Laurie Goodyear and colleagues transplanted brown fat from mice of a normal weight into mice fed with a standard or high-fat diet.

Eight to 12 weeks after transplantation, the mice fed a normal diet showed improved glucose tolerance, increased insulin sensitivity, lower body weights and decreased fat mass. Meanwhile, mice given a 'fake' transplant of glass or beads did not show any improvement, proving this way the efficacy of brown fat [13].

Also, transplantation of normal fat on mice with generalized lipodystrophy has reversed hyperglycemia, together with dramatical

\section{References}

1. Barraquer-Ferre L. Lipodystrophie progressive: syndrome de Barraquer-Simons. Press Med 1935; 43:1672.

2. Hegele RA, Joy TR, Al-Attar SA, Rutt BKJ. Thematic review series: Adipocyte Biology, Lipodystrophies: windows on adipose biology and metabolism. J Lipid Res 2007; 48(7):143344.

3. Ferrarini A, Milani D, Bottigelli M, Cagnoli G, Selicorni A. Two new cases of BarraquerSimons syndrome. Am J Med Genet 2004; 126(A):427-429.

4. Hegele RA, Cao H, Liu DM, et al. Sequencing of the reannotated LMNB2 gene reveals novel mutations in patients with acquired partial lipodystrophy. Am J Hum Gen 2006; 79(2):383389.

5. Chandran M, Phillips SA, Ciaraldi T, Henry RR. Adiponectin: more than just another fat cell hormone? Diabetes Care 2003; 26(8):24422450.

6. Hegele RA, Cao H, Huff M. LMNA R482Q mutation in partial lipodystrophy associated with reduced plasma leptin concentration. J Clin Endocrinol Metab 2000; 3(1):39-44.

7. Huang-Doran I, Sleigh A, Rochford JJ, O'Rahilly S, Savage DB. Lipodystrophy: metabolic insights from a rare disorder. J Endocrinol 2010; 207(3):245-255. reduceding in plasma insulin levels and hepatic steatosis [14].

\section{Conclusion}

It is important to study rare diseases such as the Barraquer-Simons syndrome, because they can reveal valorous insights on human metabolism and generate treatment perspectives not only for their management, but also for the most challenging diseases of our century. An intervention of fat transplantation might be considered as a therapeutical approach for our APL patient in the future.

8. Arita $\mathrm{Y}$, Kihara S, Ouchi N, et al. Adipocytederived plasma protein adiponectin acts as a platelet-derived growth factor-BB-binding protein and regulates growth factor-induced common post receptor signal in vascular smooth muscle cell. Circulation 2002; 105:2893-2898.

9. Okamoto Y, Arita Y, Nishida M. An adipocytederived plasma protein, adiponectin, adheres to injured vascular walls. Horm Metab Res 2000; 32:47-50.

10. Lantz KA, Hart SG, Planey SL, et al. Inhibition of PTP1B by trodusquemine (MSI-1436) causes fat-specific weight loss in diet-induced obese mice. Obesity 2010; 18(8):1516-23.

11. Bradford PG. Curcumin and obesity. Biofactors 2013; 39(1):78-87.

12. Okada-Iwabu M, Yamauchi T, Iwabu M, et al. A small-molecule AdipoR agonist for type 2 diabetes and short life in obesity. Nature 2013; 503(7477):493-499.

13. Stanford KI, Roeland JW, Middelbeek KL, et al. Brown adipose tissue regulates glucose homeostasis and insulin sensitivity. $J$ Clin Invest 2013; 123(1):215-223.

14. Savage DB. Mouse models of inherited lipodystrophy. Dis Model Mech 2009; 16:97986. 